\title{
Crystallization and Dynamic Mechanical Behavior of Coir Fiber Reinforced Poly(Butylene Succinate) Biocomposites
}

\author{
Xu Yan ${ }^{1}$, Changheng Liu ${ }^{2}$, Liang Qiao ${ }^{1}$, Kaili Zhu ${ }^{2}$, Hongsheng Tan ${ }^{1, *}$, Shuhua Dong ${ }^{1}$ and Zhitao Lin ${ }^{1}$ \\ ${ }^{1}$ School of Materials Science and Engineering, Shandong University of Technology, Zibo, 255000, China \\ ${ }^{2}$ Shandong Qinghe Chemical Technology Co., Ltd., Zibo, 255000, China \\ *Corresponding Author: Hongsheng Tan. Email: hstan@sdut.edu.cn \\ Received: 25 April 2021 Accepted: 05 August 2021
}

\begin{abstract}
The crystallization behavior, crystal morphology and form, and viscoelastic behavior of poly(butylene succinate) (PBS) and coir fiber/PBS composites (CPB) were investigated by differential scanning calorimetry (DSC), polarized optical microscopy (POM), X-ray diffraction (XRD) and dynamic mechanical analysis (DMA). The results of DSC measurement show that the crystallization temperature increases with the filling of coir fibers. POM images reveal that the spherulitic size and crystallization behavior of PBS are influenced by the coir fibers in the composites. XRD curves show that the crystal form of pure PBS and CPB are remaining almost identical. In addition, the storage modulus of CPB significantly increases comparing with the pure PBS. This predicted the dimensional stability and improved load-deformation temperature. In conclusion, the addition of coir fibers has a significant effect on the thermal properties of the matrix.
\end{abstract}

\section{KEYWORDS}

Poly(butylene succinate); coir fiber; crystallization; dynamic mechanical analysis

\section{Introduction}

With the progress of science and the improvement of people's living standards, environmental pollution has become a widespread concern. The use of eco-friendly biodegradable polymers has attracted increasing interest from academia and industry worldwide as a potential solution for global environmental pollution. Functional, inexpensive, and simple to manufacture, including some biodegradability, have made plant fiber a common reinforcing material for scientists and practitioners active in the field of composite materials [1]. Researchers have composited natural fibers such as kenaf, jute, flax, and bamboo fiber with resin to obtain the composites and then characterized them. The results show that the addition of the fibers has a positive effect on the mechanical and thermal properties of the composites [2-4]. These materials can undergo environmental degradation amidst the presence of micro-organisms [5].

Poly(butylene succinate) (PBS) is an eco-friendly biodegradable polyester with good processability, biodegradability, compostability, thermal and chemical resistance [6]. Due to its excellent processing properties, PBS can be processed by injection, hot compressing, extrusion, and blowing molding methods [7]. PBS can also be processed into textile products, such as monofilament, multifilament, nonwoven fabric, flat and split yarn [8]. However, its weak mechanical properties and high price are the main 
obstacles for various end-use applications [9]. To improve the performance and reduce the cost of PBS composites, series of copolymerization, hybrid, or composite modification methods have been adopted. For copolymerization methods, PBS monomers are copolymerized with other polyester monomers, such as aromatic and aliphatic polyesters [10,11]. While for the melt-processing methods, PBS is blended as a matrix polymer with other polyesters, e.g. $[12,13]$ polyethylene terephthalate (PET), polyarylester, etc., and compounded with fillers in the molten state or at high temperatures using an internal mixer or extruder to obtain homogeneous high-performance composites. In general, the filler materials used here can be summarized as the following two categories: (1) Inorganic substance [14-16]: $\mathrm{CaCO}_{3}, \mathrm{SiO}_{2}$, montmorillonite, etc. (2) Biodegradable polymers [17-20]: Starch, natural fibers, and synthetic polymer like polylactic acid (PLA), polycaprolactone, etc.

Biocomposites consist of a biodegradable matrix polymer and a lignocellulosic material as a reinforcing filler. These lignocellulosic fillers can also be naturally degraded by micro-organisms and even play a pivotal part in degrading organic matter in the natural ecosystem. Lignocellulosic fibers, such as sisal, flax, jute, coir fiber, etc., have been extensively studied due to their easy accessibility and acceptable mechanical strength [21-23]. Coir fiber is a new type of natural fiber that can be used. In terms of the content of each component in coir fiber, the content of cellulose and lignin in coir fiber is high, while the content of hemicellulose is low. This natural fiber usually has excellent mechanical and thermal properties. However, due to the presence of a large number of hydrophilic and polar hydroxyl $(-\mathrm{OH})$ groups in natural fibers, the interface between natural fibers and hydrophobic resins is very poor, resulting in poor mechanical properties of the composites. To improve the mechanical properties of the composite, alkali treatment modification of plant fibers is usually used before compounding to improve adhesion between the hydrophilic fibers and hydrophobic matrix [24-26]. The usage of coupling agents can further enhance the performance of biocomposites [27]. For instance, Calabia et al. [28] investigated that the use of silanes could improve the mechanical properties of cotton fiber-reinforced PBS composites. Lee et al. [29] studied the effects of lysine-based diisocyanate as a coupling agent on the properties of bamboo fiber/poly(lactic acid) (PLA)/PBS biocomposite. Xie et al. [30] reported the recent progress in using silane coupling agents for natural fiber/ polymer composites.

Although previous researchers have done a great deal of work, the research on the crystallization and viscoelastic behavior of coir fiber reinforced poly (butylene succinate) (PBS) biocomposites is very limited. Thus, the objective of this work is to investigate the crystallization and viscoelastic behavior of coir fiber/PBS (CPB). The materials were studied by DSC, DMA, XRD and POM.

\section{Experimental}

\subsection{Materials}

The coir fibers used in this study were produced in Vietnam. PBS (No. HX-Z1014) in dry-pellet form was supplied by the Anqing Hexing Chemical Corporation Limited (China), with a melt mass flow rate of $7.15 \mathrm{~g} / 10 \mathrm{~min}\left(125^{\circ} \mathrm{C}, 0.352 \mathrm{~kg}\right)$. Maleic anhydride $(\mathrm{MAH})$ was provided by Jinan Hongju Chenmical Reagent Factory (Jinan, China). Dicumyl peroxide (DCP) used as the initiator of grafting reaction was provide from Sinopharm Chemical Reagent Co., Ltd. (Shanghai, China).

\subsection{Preparation of CPB Composites}

\subsubsection{Pretreatment of Coir Fiber}

Before using coir fibers, the surface of the coir fibers should be treated by mercerizing. The specific treatment methods are as follows: (1) Select coir fibers with fewer impurities, and then wash them with clean water and dry them for standby. (2) Soak the dried coir fibers in $4 \% \mathrm{NaOH}$ solution for $15 \mathrm{~h}$, and then wash the alkali-treated coir fibers to neutral with distilled water and dry them at $80^{\circ} \mathrm{C}$ for $24 \mathrm{~h}$. (3) Cut the treated coir fibers to about $4 \mathrm{~mm}$ for standby. 


\subsubsection{Preparation of Compatibilizer}

The PBS resin pellets were dried at $60^{\circ} \mathrm{C}$ for $12 \mathrm{~h}$, and a certain amount of MAH and DCP were ground into powder in a mortar. The specific reaction was as follows: Heat the torque rheometer to $180^{\circ} \mathrm{C}$, and after reaching the set temperature, add PBS resin, MAH and DCP in the ratio of 97.25:2:0.75, and they were mixed at $30 \mathrm{r} / \mathrm{min}$ for $5 \mathrm{~min}$ for grafting reaction, and finally, MAH-g-PBS with a grafting rate of about $7 \%$ was obtained. After mixing, take out the graft and crush it for standby.

\subsubsection{Preparation of Composite Materials}

The flow diagram of the preparation process of coir fiber/PBS biocomposites (CPB) is shown in Fig. 1. The specific operation steps are as follows: The pretreated coir fibers and PBS were mixed according to the formula proportion. The mixture was mixed by the torque rheometer, and the samples were cut into blocks of about $4 \mathrm{~mm}$ after blending. Finally, the materials were made by injection molding, and their properties were tested. The composition of the specimens can be seen in Table 1.

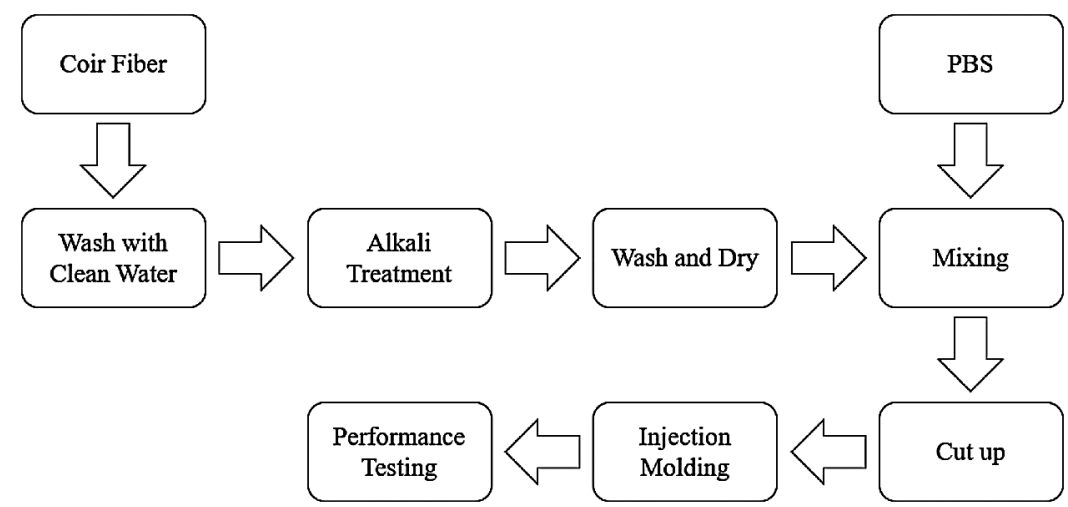

Figure 1: The flow diagram of preparing CPB

Table 1: The composition of every specimen

\begin{tabular}{llll}
\hline Materials & $\begin{array}{l}\text { Fiber content } \\
(\mathrm{wt} \%)\end{array}$ & $\begin{array}{l}\text { PBS content } \\
(\mathrm{wt} \%)\end{array}$ & $\begin{array}{l}\text { PBS- } g \text {-MAH } \\
\text { Content }(\mathrm{wt} \%)\end{array}$ \\
\hline PBS & 0 & 100 & 0 \\
CPB1 & 20 & 72 & 8 \\
CPB2 & 40 & 52 & 8 \\
\hline
\end{tabular}

\subsection{Measurements}

The thermal analysis was performed with a Q100-DSC (TA Instruments) differential scanning calorimeter (DSC) in nitrogen according to ISO/11357-1-2009. The dosage of each test sample was 5$10 \mathrm{mg}$. These samples were first heated to $160^{\circ} \mathrm{C}$ and held for $3 \mathrm{~min}$ to erase the previous heat history. Afterwards, they were cooled to $30^{\circ} \mathrm{C}$ and heated again to $160^{\circ} \mathrm{C}$. The heating and cooling rates were both $20^{\circ} \mathrm{C} / \mathrm{min}$. The crystallinity $\left(X_{\mathrm{c}}\right)$ of pure PBS and CPB biocomposites were calculated using DSC data with the formula below [31]:

$X_{C}=\frac{\left|\Delta H_{m-} \Delta H_{c c}\right|}{\Phi_{P B S} \Delta H_{m}^{0}} \times 100$ 
where $\Delta H_{\mathrm{m}}$ is the melting enthalpy of the sample, $\Delta H_{\mathrm{cc}}$ is the cold crystallization enthalpy of biocomposites, $\Phi_{\mathrm{PBS}}$ is the mass fraction of PBS in the biocomposites, and $\Delta H_{\mathrm{m}}^{0}$ is the theoretical melting enthalpy of PBS $(200 \mathrm{~J} / \mathrm{g})$. A is the mass ratio of coir fibers.

Wide angle X-ray scattering (WAXS) measurements were performed using a D8 Advance (Bruker AXS) type $\mathrm{X}$-ray diffractometer with a $\mathrm{Cu} \mathrm{K} \alpha$ radiation Ni-filter and scintillation counter as a detector at $40 \mathrm{kV}$ and $23 \mathrm{~mA}$. The samples were scanned at a scanning speed of $2^{\circ}$ per min in the continuous mode with a step size of $0.02^{\circ}$ and a diffraction angle $2 \theta$ in the range of $5-60^{\circ}$ range.

The crystallite morphology of $\mathrm{CPB}$ at $90^{\circ} \mathrm{C}$ was observed by a polarized optical microscope (POM, XPV-203E, Shanghai Changfang Optical Instrument Co., Ltd., China) with a hot stage. The samples were placed on the heating stage of the microscope. They were then heated to $140^{\circ} \mathrm{C}$ at a rate of $20^{\circ} \mathrm{C} / \mathrm{min}$ and maintained for $5 \mathrm{~min}$ to eliminate the thermal history. After that, the samples were cooled to $90^{\circ} \mathrm{C}$ by air. Finally, the temperature was maintained, and the micrographs of POM were obtained.

The viscoelastic analysis of the specimens was studied by a dynamic mechanical analyzer (DMA, Q800-DMA TA Instrument) according to ISO/6721-1-2011. The rectangular specimen had dimensions of $60 \times 9.95 \times 4 \mathrm{~mm}$. The samples were first cooled to $-150^{\circ} \mathrm{C}$ with liquid nitrogen and then heated to $100^{\circ} \mathrm{C}$ at a rate of $5{ }^{\circ} \mathrm{C} / \mathrm{min}$ with a frequency of $1 \mathrm{~Hz}$. The loss factor $(\tan \delta)$ and storage modulus $\left(E^{\prime}\right)$ data were measured.

\section{Results and Discussion}

\subsection{DSC Analysis}

The DSC cooling and second heating curves of three samples with different coir fiber contents are shown in Figs. 2a and 2b. The detail results are listed in Table 2. As shown in Fig. 2a, the crystallization temperatures $\left(T_{\mathrm{c}}\right)$ of the PBS composites with 0 (Pure PBS), $20 \mathrm{wt} \%$ (CPB1), $40 \mathrm{wt} \%$ (CPB2) coir fibers are $57.8^{\circ} \mathrm{C}, 62.8^{\circ} \mathrm{C}, 65.6^{\circ} \mathrm{C}$ when cooling from the melt, respectively. Thus, the $T_{\mathrm{c}}$ of the composites increases, and the melt crystallizes faster when more coir fibers are added. This indicates that coir fibers act as nucleating agents and accelerates the crystallization process of PBS. As shown in Fig. 2b, the melting temperatures $\left(T_{\mathrm{m}}\right)$ of the composites are similar to that of pure PBS $\left(103.0^{\circ} \mathrm{C}\right)$ during the melting process, it should be noticed that an exothermic peak $\left(T_{\text {exo }}\right)$ appears before $T_{\mathrm{m}}$ in each sample, which may be caused by recrystallization. A similar phenomenon has been reported by Yasuniwa et al. [32] and Zhan et al. [33]. In their study, the recrystallization peak of pure PBS shifted to a higher temperature by slowing down the cooling process. They attributed this to the "annealing peak" characteristic of isothermally crystallized PBS. In this work, it is observed that the $T_{\text {exo }}$ shifted to a higher temperature with increasing coir fibers content, meaning that the addition of coir fibers can improve the crystallization of biocomposites.

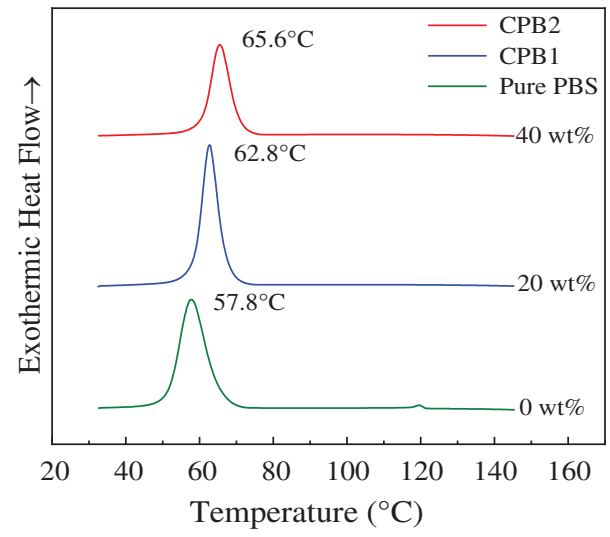

(a)

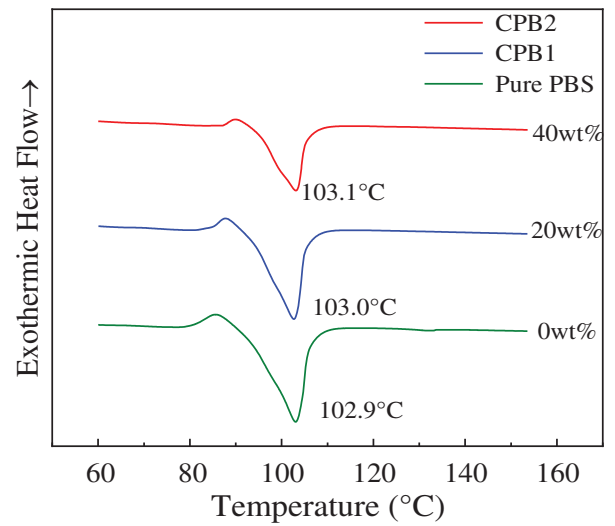

(b)

Figure 2: DSC curves (a) Cooling curves and (b) Second heating curves 
Table 2: $T_{\mathrm{m}}, T_{\mathrm{c}}, T_{\mathrm{exo}}, \Delta H_{\mathrm{m}}$, and $X_{\mathrm{c}}$ of PBS, CPB1 and CPB2

\begin{tabular}{lllllll}
\hline Materials & $T_{\mathrm{m}}\left({ }^{\circ} \mathrm{C}\right)$ & $T_{\mathrm{c}}\left({ }^{\circ} \mathrm{C}\right)$ & $T_{\text {exo }}\left({ }^{\circ} \mathrm{C}\right)$ & $\Delta H_{\mathrm{cc}}(\mathrm{J} / \mathrm{g})$ & $\Delta H_{\mathrm{m}}(\mathrm{J} / \mathrm{g})$ & $X_{\mathrm{c}}(\%)$ \\
\hline PBS & 57.8 & 103.0 & 85.5 & 4.0 & 50.2 & 23.1 \\
CPB1 & 62.8 & 102.9 & 87.9 & 4.4 & 45.5 & 28.4 \\
CPB2 & 65.6 & 103.1 & 90.0 & 2.4 & 31.3 & 26.1 \\
\hline
\end{tabular}

The $X_{\mathrm{c}}$ of the biocomposites (CPB1, 28.4\%; CPB2, 26.1\%) is higher than that of pure PBS (23.1\%). This indicates that the addition of coir fibers promotes the crystallization of PBS. CPB1 has the highest $X_{\mathrm{c}}$, and $X_{\mathrm{c}}$ decreases with the further increase in the amount of coir fibers. This suggests that a high amount of coir fibers might hinder the crystallization of PBS. A similar investigation was carried out by Liang et al. [34] on Kenaf fiber/PBS biocomposites. They found that the crystallization enthalpy of PBS/KF composites decreased with the increase of KF content when KF content exceeded 20\%, which indicates that the existence of a large number of fibers may hinder the resin crystallization.

\subsection{Spherulitic Morphology and Crystal Form}

To investigate the effect of coir fibers on the crystallization process, POM was used. Fig. 3 shows the isothermal crystallization behavior of pure PBS and CPB biocomposites at $90^{\circ} \mathrm{C}$. The coir fibers could be observed throughout the process in both CPB1 and CPB2, and they occupied large spaces. As for PBS, at the beginning ( $3 \mathrm{~min}$ ), few crystals could be observed. As time progressed, more and more crystals appeared and grew bigger. At around $80 \mathrm{~min}$, the screen was almost filled with crystals, and the crystal growth of PBS stopped. During the whole process, new crystals kept appearing. Therefore, the size of crystals in PBS was a wide range. On the other hand, the crystallization process took much less time for CPB1 and CPB2. At $3 \mathrm{~min}$, a large number of small spherulites appeared in CPB1, while more spherulites appeared in CPB2. This indicates the nucleating agent effect of coir fibers, which provided heterogeneous nuclei. Then, these small crystals grew bigger gradually. After $20 \mathrm{~min}$, the area without coir fibers was almost filled with similar-sized spherulites. Finally, the crystals of CPB1 and $\mathrm{CPB} 2$ stopped growing.

(a)
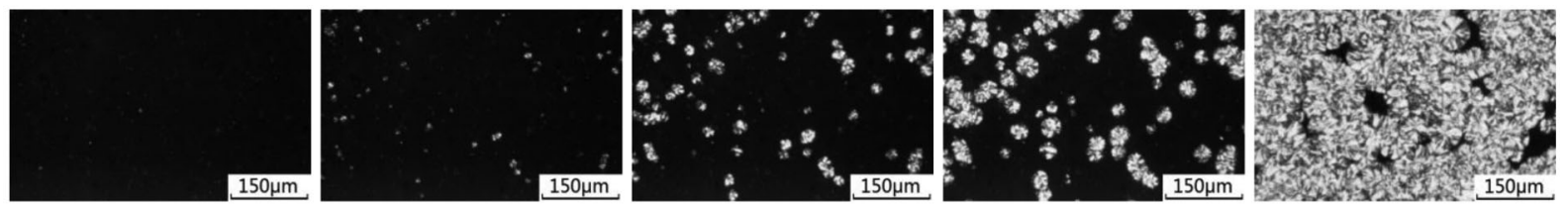

(b)
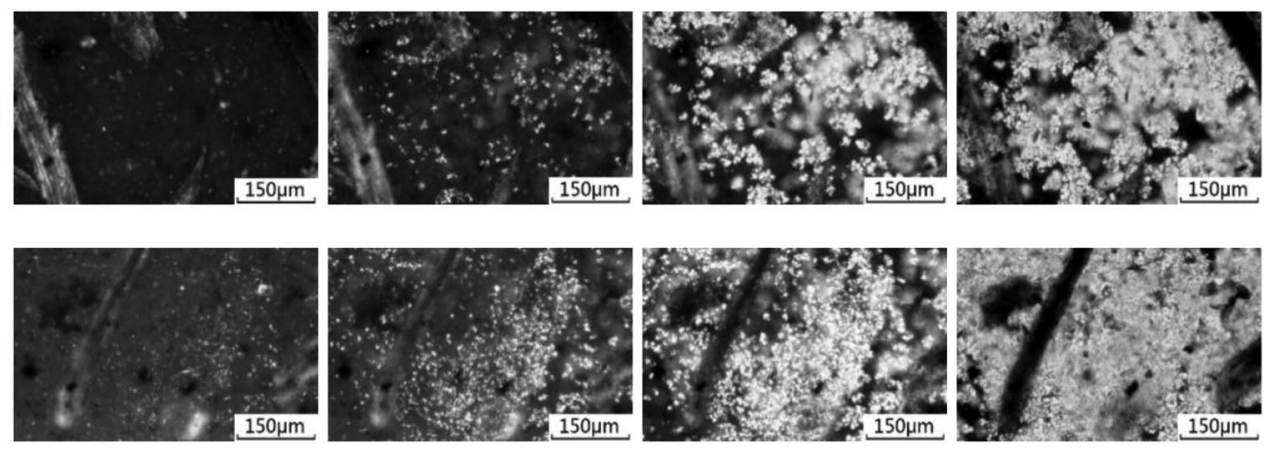

$(3 \mathrm{~min})$

$(6 \mathrm{~min})$

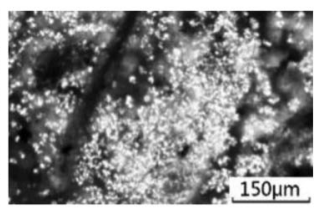

(12min)

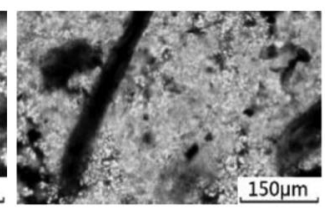

(20min)
$(80 \mathrm{~min})$

Figure 3: POM micrographs at $90^{\circ} \mathrm{C}$ for 3, 6, 12, 20 and 80 min (a) PBS, (b) CPB1 and (c) CPB2 
The five average diameters ( $\mathrm{r}$ ) of the crystals in the samples for each time are recorded and shown in Fig. 4. Initially, the crystal growth rate of CPB1 and CPB2 was much higher than that of pure PBS. However, space limits the growth of crystals. When the time reached around $20 \mathrm{~min}$, the growth of the spherulites of CPB1 and CPB2 gradually slowed down, and then the diameters of these two samples were maintained at those sizes. The final average crystal size of CPB1 $(26.9 \mu \mathrm{m})$ was higher than that of CPB2 $(22.8 \mu \mathrm{m})$. This is due to that CPB1 $(20 \mathrm{wt} \%)$ has less fiber content than CPB2 $(40 \mathrm{wt} \%)$. Then, there was more space for the crystal growth in CPB1, where the crystals eventually reached larger sizes.

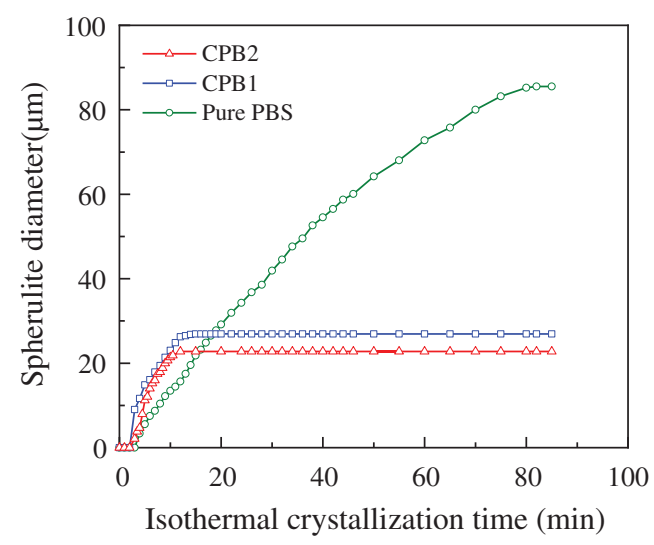

Figure 4: Spherulite diameters of $v s$. time during isothermal crystallization at $90{ }^{\circ} \mathrm{C}$

WAXS patterns of pure PBS, CPB1 and CPB2 can be found in Fig. 5. The WAXS pattern of PBS shows two sharp peaks locate at $19.6^{\circ}, 22.6^{\circ}$, and a weak peak $\left(21.9^{\circ}\right)$ saddles on one side of the latter peak. These peaks can be indexed as the (020), (021) and (110) planes of the $\alpha$ crystal form [34] of PBS, respectively. The WAXS patterns of CPB1 and CPB2 are almost identical to the shapes and peak positions of pure PBS, which suggests that the coir fibers have little effect on the crystal form of PBS, though it dramatically accelerates the crystallization process.

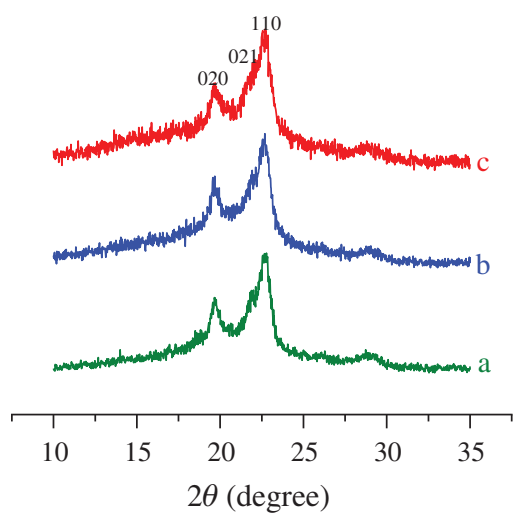

Figure 5: WAXS curves (a) Pure PBS, (b) CPB1 and (c) CPB2

\subsection{Dynamic Mechanical Analysis}

The loss factor $(\tan \delta)$ is the ratio between loss modulus $\left(E^{\prime \prime}\right)$ and storage modulus $\left(E^{\prime}\right)$. The $\tan \delta$ of the composites affects the interactions between matrix/fiber and weak adhesion if the value of $\tan \delta$ is high [35]. As shown in Fig. $6 a$, the main $\tan \delta$ peaks appear at $-12.63^{\circ} \mathrm{C},-12.24^{\circ} \mathrm{C}$ and $-12.82^{\circ} \mathrm{C}$, respectively, 
corresponding to the glass transition temperatures $\left(T_{\mathrm{g}}\right)$ of PBS, CPB1 and CPB2. This result indicates that $T_{\mathrm{g}}$ can be barely affected by the inclusion of coir fibers. The $\tan \delta$ peak height is generally used to assess the damping ability of the composites. The peak value of $\tan \delta$ of pure PBS is 0.136 . In contrast, the $\tan \delta$ peak of the composite decreases with the addition of coir fibers, which is due to the interfacial bonding between the fibers and the PBS matrix, which hinders molecular motion. When the amount of coir fibers increases, the peak value of $\tan \delta$ continued to decrease.

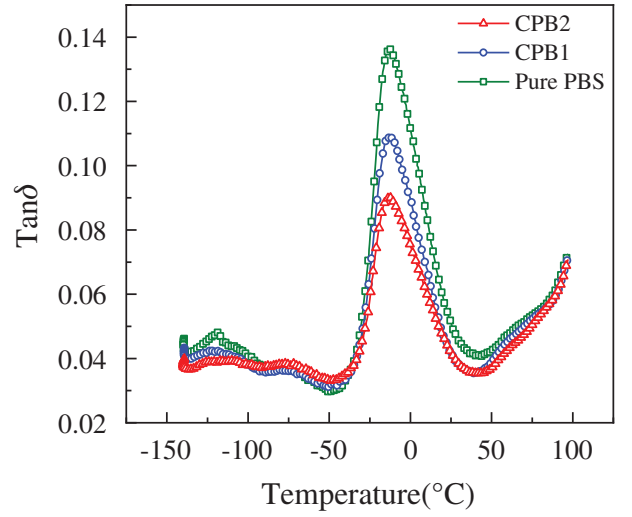

(a)

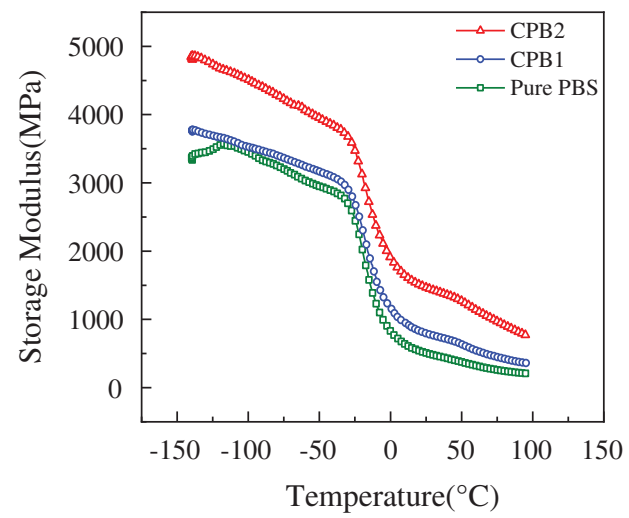

(b)

Figure 6: Dynamic viscoelastic spectrum (a) Loss factor $(\tan \delta)$ and (b) Storage modulus $\left(E^{\prime}\right)$ curves

$E^{\prime}$ is the ability of the material to resist deformation. The higher the $E^{\prime}$ is, the more rigid the material and the weaker the deformation capacity. The values of $E^{\prime}$ are closely related to the mobility of polymer molecular chains and side groups [36]. From Fig. 6b, $E^{\prime}$ increases with the rising number of coir fibers amount. This phenomenon indicates that the ability of the composites to resist deformation is improved. At $0^{\circ} \mathrm{C}$, the $E^{\prime}$ of PBS is $823 \mathrm{MPa}$, while the $E^{\prime}$ of the composite is $1169 \mathrm{MPa}$ when the fiber content is $20 \mathrm{wt} \%$. When the fiber content is further increased to $40 \mathrm{wt} \%$, the $E^{\prime}$ of the composite is $1900 \mathrm{MPa}$, which is $130.86 \%$ higher than that of the pure PBS. The results show that the deformation resistance of the material is significantly improved by adding coir fibers. The higher fiber content, the higher the $E^{\prime}$. When the temperature is $90^{\circ} \mathrm{C}$, the $E^{\prime}$ of PBS, CPB1 and CPB2 are 218, 337 and $819 \mathrm{MPa}$, respectively, indicating that $\mathrm{CPB} 2$ has better resistance to deformation than PBS and CPB1 at higher temperatures.

\section{Conclusions}

Coir fiber reinforced PBS composites were prepared by a torque rheometer and injection molding machine. During the non-isothermal/isothermal crystallization process, it is found that the crystallization rate of PBS is faster with the addition of coir fibers, but the presence of too many fibers also limited the crystallization of PBS. Moreover, the crystal size shows a negative correlation with the fiber content. The XRD results show that the addition of coir fibers only promoted the crystallization of PBS and does not change its crystal morphology. The DMA analysis shows that the storage modulus of CPB2 is higher than that of pure PBS and CPB1 in the text temperature range, which indicated that the deformation resistance of $\mathrm{CPB} 2$ biocomposites is improved. Overall, it can be concluded that $\mathrm{CPB}$ has more satisfactory properties when the fiber content is $40 \%$ compared to other contents. Therefore, it is suitable for further research and commercialization as a promising green cohesive material.

Funding Statement: The Natural Science Foundation of Shandong Province, China (Grant No. ZR2020QE075). 
Conflicts of Interest: The authors declare that they have no conflicts of interest to report regarding the present study.

\section{References}

1. Singh, S. K., Sagar, M. K., Upadhyay, R. K. (2021). Mechanical characterization of plant fortified fiber polymer composites. Materials Today: Proceedings (in Press).

2. Asim, M., Jawaid, M., Nasir, M., Saba, N. (2018). Effect of fiber loadings and treatment on dynamic mechanical, thermal and flammability properties of pineapple leaf fiber and kenaf phenolic composites. Journal of Renewable Materials, 6(4), 383-393. DOI 10.7569/JRM.2017.634162.

3. Safwan, A., Jawaid, M., Sultan, M. T. H., Hassan, A. (2018). Preliminary study on tensile and impact properties of kenaf/bamboo fiber reinforced epoxy composites. Journal of Renewable Materials, 6(5), 529-535. DOI 10.7569/ JRM.2018.634103.

4. Khan, T., Sultan, M. T. H., Jawaid, M., Safri, S. N. A., Shah, A. U. M. et al. (2021). The effects of stacking sequence on dynamic mechanical properties and thermal degradation of kenaf/jute hybrid composites. Journal of Renewable Materials, 9(1), 73-84. DOI 10.32604/jrm.2021.011385.

5. Kim, H. S., Kim, H. J., Lee, J. W., Choi, I. G. (2006). Biodegradability of bio-flour filled biodegradable poly (butylene succinate) bio-composites in natural and compost soil. Polymer Degradation \& Stability, 91(5), 1117-1127. DOI 10.1016/j.polymdegradstab.2005.07.002.

6. Xu, J., Guo, B. (2010). Poly (butylene succinate) and its copolymers: Research, development and industrialization. Biotechnology Journal, 5(11), 1149-1163. DOI 10.1002/biot.201000136.

7. Fujimaki, T. (1998). Processability and properties of aliphatic polyesters, 'BIONOLLE', synthesized by polycondensation reaction. Polymer Degradation and Stability, 59(1-3), 209-214. DOI 10.1016/S0141-3910 (97)00220-6.

8. Okamoto, K., Ray, S. S., Okamoto, M. (2003). New poly (butylene succinate)/layered silicate nanocomposites. II. Effect of organically modified layered silicates on structure, properties, melt rheology, and biodegradability. Journal of Polymer Science Part B: Polymer Physics, 41(24), 3160-3172. DOI 10.1002/(ISSN)1099-0488.

9. Feng, Y. H., Shen, H. Z., Qu, J. P., Liu, B., He, L. Y. et al. (2011). Preparation and properties of pbs/sisal-fiber composites. Polymer Engineering \& Science, 51(3), 474-481. DOI 10.1002/pen.21852.

10. Deng, L. M., Wang, Y. Z., Yang, K. K., Wang, X. L., Zhou, Q. et al. (2004). A new biodegradable copolyester poly (butylene succinate-co-ethylene succinate-co-ethylene terephthalate). Acta Materialia, 52(20), 5871-5878. DOI 10.1016/j.actamat.2004.09.002.

11. Chen, C. H., Peng, J. S., Chen, M., Lu, H. Y., Tsai, C. J. et al. (2009). Synthesis and characterization of poly (ethylene succinate) and its copolyesters containing minor amounts of butylene succinate. Journal of Applied Polymer Science, 111(3), 1433-1439. DOI 10.1002/app.29035.

12. Gan, Z., Abe, H., Kurokawa, H., Doi, Y. (2001). Solid-state microstructures, thermal properties, and crystallization of biodegradable poly (butylene succinate) (PBS) and its copolyesters. Biomacromolecules, 2(2), 605-613. DOI $10.1021 / \mathrm{bm} 015535 \mathrm{e}$.

13. Dubois, P., Jacobs, C., Jérôme, R., Teyssie, P. (1991). Macromolecular engineering of polylactones and polylactides. 4. Mechanism and kinetics of lactide homopolymerization by aluminum isopropoxide. Macromolecules, 24(9), 2266-2270. DOI 10.1021/ma00009a022.

14. Suhartini, M., Mitomo, H., Yoshii, F., Nagasawa, N., Kume, T. (2001). Radiation crosslinking of poly (butylene succinate) in the presence of inorganic material and its biodegradability. Journal of Polymers \& the Environment, 9(4), 163-171. DOI 10.1023/A:1020417422032.

15. Ray, S. S., Okamoto, K., Okamoto, M. (2003). Structure-property relationship in biodegradable poly (butylene succinate)/layered silicate nanocomposites. Macromolecules, 36(7), 2355-2367. DOI 10.1021/ma021728y.

16. Ma, P., Wang, X., Liu, B., Li, Y., Chen, S. H. et al. (2012). Preparation and foaming extrusion behavior of polylactide acid/polybutylene succinate/montmorillonoid nanocomposite. Journal of Cellular Plastic, 48(2), 191-205. DOI 10.1177/0021955X11434182. 
17. Lai, S. M., Huang, C. K., Shen, H. F. (2005). Preparation and properties of biodegradable poly (butylene succinate)/starch blends. Journal of Applied Polymer Science, 97(1), 257-264. DOI 10.1002/(ISSN)1097-4628.

18. Ohkita, T., Lee, S. H. (2010). Crystallization behavior of poly(butylene succinate)/corn starch biodegradable composite. Journal of Applied Polymer Science, 97(3), 1107-1114. DOI 10.1002/app.21741.

19. Li, J., Yong, H., Inoue, Y. (2003). Thermal and mechanical properties of biodegradable blends of poly(l-lactic acid) and lignin. Polymer International, 52(6), 949-955. DOI 10.1002/pi.1137.

20. Qiu, Z., Ikehara, T., Nishi, T. (2003). Miscibility and crystallization behaviour of biodegradable blends of two aliphatic polyesters. poly (3-hydroxybutyrate-co-hydroxyvalerate) and poly (butylene succinate) blends. Polymer, 44(24), 7519-7527. DOI 10.1016/j.polymer.2003.09.029.

21. Feng, Y. H., Zhang, D. W., Qu, J. P., He, H. Z., Xu, B. P. (2011). Rheological properties of sisal fiber/poly (butylene succinate) composites. Polymer Testing, 30(1), 124-130. DOI 10.1016/j.polymertesting.2010.11.004.

22. Liu, L. F., Yu, J. Y., Cheng, L. D., Qu, W. W. (2009). Mechanical properties of poly (butylene succinate) (PBS) biocomposites reinforced with surface modified jute fibre. Composites Part A: Applied Science and Manufacturing, 40(5), 669-674. DOI 10.1016/j.compositesa.2009.03.002.

23. Dorez, G., Taguet, A., Ferry, L., Lopez-Cuesta, J. M. (2013). Thermal and fire behavior of natural fibers/pbs biocomposites. Polymer Degradation \& Stability, 98(1), 87-95. DOI 10.1016/j.polymdegradstab.2012.10.026.

24. Joseph, S., Joseph, K., Thomas, S. (2006). Green composites from natural rubber and oil palm fiber: Physical and mechanical properties. International Journal of Polymeric Materials \& Polymeric Biomaterials, 55(11), 925-945. DOI 10.1080/00914030600550505.

25. Renreng, I., Bakri, B., Chandrabakty, S. (2021). Evaluation of mechanical and physical properties of pressed coir fiber/epoxy composite with $\mathrm{NaOH}$ and microwave treatment of fiber. Journal of Renewable Materials, 9(2), 325335. DOI 10.32604/jrm.2021.012774.

26. van de Weyenberg, I., Truong, T. C., Vangrimde, B., Verpoest, I. (2006). Improving the properties of UD flax fibre reinforced composites by applying an alkaline fibre treatment. Composites Part A: Applied Science \& Manufacturing, 37(9), 1368-1376. DOI 10.1016/j.compositesa.2005.08.016.

27. John, M. J., Anandjiwala, R. D. (2008). Recent developments in chemical modification and characterization of natural fiber-reinforced composites. Polymer Composites, 29(2), 187-207. DOI 10.1002/(ISSN)1548-0569.

28. Calabia, B., Ninomiya, F., Yagi, H., Oishi, A., Taguchi, K. et al. (2013). Biodegradable poly (butylene succinate) composites reinforced by cotton fiber with silane coupling agent. Polymers, 5(1), 128-141. DOI 10.3390/ polym5010128.

29. Lee, S. H., Wang, S. (2006). Biodegradable polymers/bamboo fiber biocomposite with bio-based coupling agent. Composites Part A: Applied Science and Manufacturing, 37(1), 80-91. DOI 10.1016/j.compositesa.2005.04.015.

30. Xie, Y. J., Hill, C. A. S., Xiao, Z. F., Militz, H., Mai, C. (2010). Silane coupling agents used for natural fiber/ polymer composites: A review. Composites Part A: Applied Science and Manufacturing, 41(7), 806-819. DOI 10.1016/j.compositesa.2010.03.005.

31. Luo, Q., Wang, M., Zhang, H., Ouyang, Y., Lin, H. et al. (2021). Preparation and properties of bio-based flame retardant L-APP/poly (L-lactic acid) composites. Journal of Renewable Materials, 9(12), 2067-2076. DOI $10.32604 / \mathrm{jrm} .2021 .016255$.

32. Yasuniwa, M., Satou, T. (2002). Multiple melting behavior of poly (butylene succinate). I. Thermal analysis of melt-crystallized samples. Journal of Polymer Science Part B: Polymer Physics, 40(21), 2411-2420. DOI 10.1002/(ISSN)1099-0488.

33. Zhan, M. Q., Chen, G. Y., Wei, Z. Y., Shi, Y. M., Zhang, W. X. (2013). Nonisothermal crystallization and morphology of poly (butylene succinate)/layered double hydroxide nanocomposites. Chinese Journal of Polymer Science, 31(1), 187-200. DOI 10.1007/s10118-013-1200-4.

34. Liang, Z. C., Pan, P. J., Zhu, B., Dong, T., Inoue, Y. (2010). Mechanical and thermal properties of poly (butylene succinate)/plant fiber biodegradable composite. Journal of Applied Polymer Science, 115(6), 3559-3567. DOI 10.1002/app.29848. 
35. Neto, J. S. S., Lima, R. A. A., Cavalcanti, D. K. K., Souza, J. P. B., Aguiar, R. A. A. et al. (2018). Effect of chemical treatment on the thermal properties of hybrid natural fiber-reinforced composites. Journal of Applied Polymer Science, 136(10), 47154. DOI 10.1002/app.47154.

36. Deák, T., Czigány, T., Tamás, P., Németh, C. (2010). Enhancement of interfacial properties of basalt fiber reinforced nylon 6 matrix composites with silane coupling agents. Express Polymer Letters, 4(10), 590-598. DOI 10.3144/expresspolymlett.2010.74. 